\title{
Body Image Perception and Self-Esteem among University Students in Cairo
}

\author{
${ }^{1}$ Manar M. Keshk; ${ }^{2}$ Hoda I. Fahim; ${ }^{2}$ Azza M. Hassan; ${ }^{2}$ Dina N. K. Boulos \\ ${ }^{1}$ Ministry of Health and Population, Preventive Sector \\ ${ }^{2}$ Department of Community, Environmental and Occupational Medicine, Faculty of \\ Medicine, Ain Shams University.
}

Received: June, $2018 \quad$ Accepted: October, 2018

\begin{abstract}
Introduction: Body image (BI) is a multidimensional concept, with behavioral, psychological and cultural components. Objectives: to describe body image perception and satisfaction among university students, to identify factors affecting body image perception and satisfaction and to determine the relation between body image perception, satisfaction, and self-esteem. Methods: A cross sectional study was conducted among 400 university students from Ain shams university and Misr University for Science and Technology (MUST). Self-administered questionnaire was used including questions about socio-demographic factors like age and gender, body image figure scale to measure body image perception and satisfaction, scale to measure body image concerns and Rosenberg scale of self-esteem. Body weight and height were also measured to calculate BMI. Results: 400 students participated in the study, 206 (51.5\%) from Ain Shams University and $194(48.5 \%)$ from MUST University. Their mean age was $20.6 \pm 2.2$ years, 254 $(63.5 \%)$ were females. Females preferred significantly smaller figures on figure scale as their ideal male body figure and smaller body figures for their own gender they think males would prefer. Measurement of body image satisfaction showed that $221(55.25 \%)$ participants wanted to lose weight, $123(30.75 \%)$ satisfied with their body weight, and 56 (14\%) wanted to gain weight. $333(83.2 \%)$ participants had positive BI concerns and 366 (91.5\%) showed high self-esteem. There was a statistically significant positive correlation between self-esteem score and body image concerns scale. Conclusion: The majority of University students perceived their body status as normal and preferred average body shapes as their ideal for their gender and other gender. More than half of the student underestimated their body image so; innovative strategies are required to ensure better appreciation of the actual body size and understanding of the health consequences of overweight and obesity and to reverse the acceptance for larger body size.
\end{abstract}

Key words: body image, satisfaction, self-esteem, University students

Corresponding auther: Azza M. Hassan Email: drazzahassan@yahoo.com

\section{Introduction:}

Body image (BI) is defined as "a person's perceptions, thoughts, and feelings about his or her own body". ${ }^{1}$ It is a multidimensional concept, with behavioral, psychological and cultural components ${ }^{2}$. Body image perception includes perceived BI which represents the image one figures about oneself, the ideal BI which the society considers as acceptable. In addition to body image of the opposite sex (BIOS) which is the perceived body image for males, by females, or vice versa. ${ }^{3}$

Positive body image can be defined as "a person`s acceptance of his own body, and appreciation of its uniqueness and 
the functions it performs" 2 . On the other hand, negative body image or a distorted body image, is defined as "a brain condition in which the person is unable to see himself or herself accurately in the mirror; but perceives his features and body size as distorted". 4

Globally, there has been an increasing interest in body image perception, due to the serious consequences of distorted body image on physical, psychological and behavioral well-being. Strict dieting, excessive weight control, low selfesteem and self-worth may be consequences to a distorted self-body image. ${ }^{4-5}$ Positive body image perception leads to sense of social acceptance, selfconfidence, and life satisfaction, and makes easier adoption of healthier attitudes and practices regarding eating behaviors and physical activity. ${ }^{1}$

Stunkard and colleagues developed a tool illustrating a series of silhouette pictures representing different body sizes. Silhouette Show card illustrates sexspecific different body sizes arranged ascending. It is used as an easy visual tool to determine perceived body size in survey settings as well as a measure of other important concepts such as ideal body size for assessing body size dissatisfaction. ${ }^{6}$

In a study conducted among female Kuwaiti university students assessing body image perceptions using female silhouettes figures, the difference between current perceived body image (PBI) and ideal body image (IBI) was used as a measure of body image dissatisfaction (BID). Study results revealed high significant correlation within PBI, IBI, BID and between them and BMI. ${ }^{7}$ In a study involving 2203 Turkish adolescent students, aged 15-18 years old, using the Rosenberg SelfEsteem Scale (SES) body image dissatisfaction was related to low selfesteem and depression in adolescents. ${ }^{8}$ University students may be more vulnerable to different pressures to follow perceived standards of physical appearance, during a crucial age that is important for the development of one's identity in relation to physical selfevaluation and self-worth. ${ }^{9}$

A positive body image means a real perception of body size leading to a sense of wellbeing and feeling comfortable with it. On the other hand, a negative body image means a distorted perception of the body size leading to continuous comparisons with others and feeling dissatisfaction. In some cultures, satisfaction with body image may be a factor leading to maintaining a higher BMI and contribute to the prevailing overweight and obesity problem. Contrary being unhappy with own body and having a negative body image perception can lead to many detrimental effects especially among young adults. ${ }^{10}$ However, studies on body image perception conducted in the Eastern Mediterranean Region are scarce especially among university students. ${ }^{10-11}$ As cultural and environmental factors have great impact on shaping standards of ideal body size and its consequences national studies are needed. The current study aimed to describe body image perception and satisfaction among university students, to identify factors affecting body image perception and satisfaction and to determine the relation between body image perception, satisfaction, and self-esteem.

\section{Methods:}

Study Design: A cross sectional study was carried out during the academic year 2016 / 2017 including students from Ain Shams University (206 students) and Misr University for Science and Technology (MUST) (194 students) to represent governmental and private universities in Greater Cairo. Students from two faculties from each university "Faculty of medicine and faculty of commerce in Ain shams University, and faculty of medicine and faculty of 
business administration in MUST University" participated in the study to represent practical and theoretical faculties. In each of the four faculties, a list of the practical sections of the first four academic years was obtained and five practical sections were selected randomly in each faculty. The attendants of these classes were invited to participate in the study after finishing their class with response rate of about $80 \%$. More participants were invited till reaching required sample size (the obtained sample size was 400 students). Data collection was carried out two days every week; each faculty was visited four to five times to collect the data.

Sample Size: Assuming that the proportion of students satisfied with their body image $=50 \% \pm 5$ at $95 \%$ C. I, a sample size of at least 384 students was needed to conduct the study (using an Epi Info7 program). ${ }^{12}$ The actual obtained sample size was 400 students.

Study tools:Data were collected by using: (1) Self-administered questionnaire: having four sections. Section one: asking about sociodemographic factors; such as: age, gender, faculty name and grade, marital status, parents' education and occupation, some life style habits; such as: regular physical exercise, weekly consumption of fast foods, daily consumption of water (by cup). Section two: Body image figure scale (Stunkard Scale) (Figure 1) which presents nine male and nine female schematic silhouettes, ranging from the extreme thinness to extreme obesity ${ }^{6}$. The figures are numbered from one (the thinnest) to nine (the fattest). It is used to measure body image perception (where participants were asked to self-select four figures, one figure that best indicates their current body size, one figure that reflects their perception of ideal body size, one figure of the other gender that she/he thinks, is ideal and one figure of her/his gender that is thought to be preferred by the opposite sex). Assessment of estimated self-body image was done by subtracting the conventional code assigned to the actual BMI calculated for the participant from the one corresponding to her/his feel figure. The resulting score, range from -3 to +3 , negative values mean weight status underestimation, positive values mean weight status overestimation and a score of 0 means a realistic perception of one's weight status. Assessment of body image satisfaction was by subtracting the figure number that represents feel (own) body image, from number which represents a preferred body image. Negative values mean the desire to be fatter, positive values means the desire to be thinner and zero means a current body image satisfaction. Section three: scale to measure body image concerns (positive or negative body image). This scale was designed, translated to Arabic, and validated by (Abdul-Naby, 2008) based on the body image concerns (BIC) by (Cooper et al., 1987. $)^{13-14}$ It includes 27 statements that measure how frequently the participants (always, sometimes, no) feel, towards their current body image, whether their family and friends criticize (or appreciate) their current body status, if they wish to have plastic surgery, and if they wish to look like a celebrity. The mean of scores for answers of this scale is 67. The score for the answered questionnaire was compared to the mean score (67), and interpreted as follows: results less than 67 means negative body image concerns and results more than 67 means positive body image concerns. Section four: Rosenberg scale of selfesteem (Rosenberg, 1989) ${ }^{15}$. Which is a ten-item Likert scale answered with a four-point scale, from strongly agree to strongly disagree. The scale measures the state of self-esteem by asking the respondents to reflect on their current feelings. Scores of the scale range from $0-30$. Scores $\geq 15$ are within normal range; scores $<15$ suggest low self- 
esteem. (2) Measurement of body mass index "BMI": Body weight and height were measured to calculate BMI of participants according to the World Health Organization (WHO) guidelines (World Health Organization, 2004) ${ }^{16}$. Participants were weighed in kilograms by a digital weight portable scale. They were directed to stand -without wearing shoes- on the center of the scale, with hands at sides, and looking straight ahead. The scale was put on a hard surface -not carpeted -floor._The height was assessed by measuring tape, fixed to a wall, and the participant was asked to stand barefoot as straight as possible against it, BMI for participants was calculated through the formula (Bodyweight in kilograms divided by height in meters squared), the BMI results were categorized and coded as follows:

\begin{tabular}{|l|l|l|}
\hline BMI result & Category & Code \\
\hline Underweight & $<18.5$ & 1 \\
\hline Normal weight & $18.5-24,9$ & 2 \\
\hline Overweight & $25-29,9$ & 3 \\
\hline Obesity & $\geq 30$ & 4 \\
\hline
\end{tabular}

These codes were used in determining the body image estimation and body image satisfaction using the body image figure scale, as mentioned above.

\section{Data Management and Analysis:}

The collected data were revised, coded, and introduced to a PC using Microsoft excel software; which was used for data cleaning and checking for quality. Statistical package for Social Science (IBM SPSS 23 for windows) was used for performing data analysis. Descriptive statistics were presented as mean \pm standard deviation for continuous variables and frequency distribution for categorical variables. Appropriate tests of significance for comparing groups` means as Student t-test for parametric data and Mann Whitney test for nonparametric tests were performed. Chisquare test was applied in case of testing association between two categorical variables; the exact test was used instead when the expected frequency is less than 5. Pearson correlation and Spearman correlation coefficients were used to measure correlation between continuous variables. Statistical significance was set up at less than 0.05 for all analyses.

\section{Ethical consideration:}

Administrative approval was obtained from the faculty of medicine, and faculty of commerce, (Ain Shams University), as well as the faculty of medicine and faculty of business administration (MUST University). A brief introduction was given to the participants explaining the study, its objectives, and goals. All questionnaires were anonymous. All data were coded and considered confidential. The permission to use the Rosenberg scale of self-esteem was obtained online.

\section{Results:}

Total number of participants in the current study is 400 students, 206 (51.5\%) participants from Ain Shams University and 194 (48.5\%) from MUST University. Half of the study participants 204 accounting for $(51 \%)$ were medical students. Their socio-demographic data are presented in (table 1). Their age ranged from 17 to 33 years, with mean of $20.6 \pm 2.2$ years and $254(63.5 \%)$ participants were females. Most of them were Egyptians $(85.7 \%)$, Cairo residents $(86.25 \%)$, single $(92.8 \%)$ and lived with their families during the academic year $(77.5 \%)$. The majority of fathers $(82.75 \%)$ and mothers of the participants $(75.25 \%)$ were university graduates. $53.6 \%$ of fathers have professional/managerial work and $57.3 \%$ of mothers were housewives. Regarding physical activity of participants, 166 $(41.5 \%)$ participants were doing regular weekly physical exercise where most of them $(58.4 \%)$ did fitness exercises (Gym and Aerobics) (table 1). Body mass index (BMI) of participants ranged from 17.6 $\mathrm{Kg} / \mathrm{m}^{2}$ to $47.3 \mathrm{Kg} / \mathrm{m}^{2}$, with mean of $25.2 \pm 1 \mathrm{Kg} / \mathrm{m}^{2}$. Regarding their dietary 
Table (1): Socio-demographic data of participants:

\begin{tabular}{|c|c|c|c|}
\hline \multicolumn{2}{|c|}{ Participants Characteristics } & \multicolumn{2}{|c|}{ N. $=400$} \\
\hline \multirow{2}{*}{ Age (years) } & Mean \pm SD & Min & Max \\
\hline & $20.6 \pm 2.2$ & 17 & 33 \\
\hline & & $\mathrm{N}$. & $\%$ \\
\hline \multirow{2}{*}{ Gender } & Male & 146 & $36.5 \%$ \\
\hline & Female & 254 & $63.5 \%$ \\
\hline \multirow{2}{*}{ Nationality } & Egyptian & 343 & $85.75 \%$ \\
\hline & Other nationalities & 57 & $14.5 \%$ \\
\hline \multirow{2}{*}{ Residence } & Cairo & 345 & $86.25 \%$ \\
\hline & Other governorates & 50 & $13.8 \%$ \\
\hline \multirow{4}{*}{$\begin{array}{l}\text { Marital } \\
\text { status }\end{array}$} & Single & 371 & $92.8 \%$ \\
\hline & Engaged & 18 & $4.5 \%$ \\
\hline & Married & 7 & $1.8 \%$ \\
\hline & Divorced & 4 & $1.0 \%$ \\
\hline \multirow{4}{*}{$\begin{array}{l}\text { Living } \\
\text { during } \\
\text { scholar } \\
\text { year }\end{array}$} & With family & 310 & $77.5 \%$ \\
\hline & With relatives & 11 & $2.8 \%$ \\
\hline & With friends/hostel & 54 & $13.5 \%$ \\
\hline & Alone & 25 & $6.3 \%$ \\
\hline \multirow{2}{*}{$\begin{array}{l}\text { Father`s } \\
\text { education }\end{array}$} & $\begin{array}{ll}\begin{array}{l}\text { Below } \\
\text { degree }\end{array} & \text { university } \\
\end{array}$ & 69 & $17.25 \%$ \\
\hline & $\begin{array}{l}\text { University and post } \\
\text { graduate degree }\end{array}$ & 331 & $82.75 \%$ \\
\hline \multirow{4}{*}{$\begin{array}{l}\text { Father`s } \\
\text { job } \\
(\mathrm{N} .=392)\end{array}$} & Retired & 23 & $7.8 \%$ \\
\hline & $\begin{array}{ll}\text { Specialized manual } \\
\text { jobs }\end{array}$ & 12 & $3.1 \%$ \\
\hline & $\begin{array}{l}\text { Specialized non manual } \\
\text { jobs }\end{array}$ & 147 & $37.5 \%$ \\
\hline & Professional/managerial & 210 & $53.6 \%$ \\
\hline \multirow{2}{*}{$\begin{array}{l}\text { Mother`s } \\
\text { education }\end{array}$} & $\begin{array}{ll}\text { Below } & \text { university } \\
\text { degree } & \\
\end{array}$ & 99 & $24.75 \%$ \\
\hline & $\begin{array}{l}\text { University and post } \\
\text { graduate degree }\end{array}$ & 301 & $75.25 \%$ \\
\hline \multirow{3}{*}{$\begin{array}{l}\text { Mother's } \\
\text { job }\end{array}$} & Housewife & 229 & $57.3 \%$ \\
\hline & $\begin{array}{l}\text { Specialized non manual } \\
\text { jobs }\end{array}$ & 105 & $26.3 \%$ \\
\hline & Professional/managerial & 66 & $16.5 \%$ \\
\hline \multicolumn{2}{|c|}{ Regular physical activity } & 166 & $41.5 \%$ \\
\hline \multirow{5}{*}{$\begin{array}{l}\text { Type of } \\
\text { physical } \\
\text { exercise } \\
\mathrm{N}=166\end{array}$} & Fitness & 97 & $58.4 \%$ \\
\hline & Football & 25 & $15.1 \%$ \\
\hline & Swimming & 15 & $9 \%$ \\
\hline & Walking & 15 & $9 \%$ \\
\hline & Other* & 14 & $8.5 \%$ \\
\hline
\end{tabular}

habits, most of them $(93.5 \%)$ consumed junk foods, at least once weekly. The mean number of weekly junk meals consumed was $4 \pm 3$ meals/week. The mean of daily water drinking of the participants was $6 \pm 4$ cups/day. More than half of study participants $(53.2 \%)$ followed a weight control diet to lose weight at least once during the preceding year. Most of the study participants (79\%) followed this diet based on a personal decision and only $9.8 \%$ stated that this was based on a physician decision. More than half of the participants on diet (55.4\%) were satisfied with their diet results (table 2). (Table 3) shows distribution of participants' choices on body figure scale where $31.5 \%$ of participants selected figure 4 to represent their feeling body figure, $38 \%$ selected figure 3 to represent their ideal body figure, $45.5 \%$ selected figure 4 as the ideal body figure for other gender and $36.5 \%$ selected figure 4 as the ideal figure for their same gender in other gender`s opinion. Regarding distribution of body figures choices of the participants by their gender, (table 4) shows that females preferred significantly smaller figures as their ideal male body figure and smaller body figures for their own gender they think males would prefer.

Feeling-Actual Index (FAI) results for all participants ranged from -0.2 to 1 . The mean FAI equals $-0.6 \pm 0.7 .214(53.5 \%)$ participants tended to underestimate their own body status (selected feeling body figures less than their actual body status by BMI). 181 (45.25\%) participants had right body image estimation (selected as same feel figures as their actual BMI), 5 $(1.25 \%)$ participants overestimated their body status (selected bigger feel figures than their actual body status) (table 5). There was no statistically significant relation between participants' FAI scores and their different characteristics ( $p$ value $>0.05$ ) except for BMI where normal weight participants had significantly higher scores than overweight/obese ( $p$ value < 0.05) (table 6).

(Table 7) shows participants` body image satisfaction by feeling ideal discrepancy index (FID). Score for all participants ranged from (-3 to 5) with mean of $0.68 \pm 1.2$. More than half of the participants (221) (55.25\%) wanted to lose weight, $123(30.75 \%)$ were satisfied with their current body weight, and 56 $(14 \%)$ wanted to gain more weight. Regarding relation between participants' body image satisfaction (FID score) and their different characteristics (table 8) 
Table (2): BMI and dietary habits of the participants:

\begin{tabular}{|c|c|c|c|}
\hline \multicolumn{2}{|l|}{ Participants characteristics } & \multicolumn{2}{|l|}{ N. $=400$} \\
\hline & Mean \pm SD & Minimum & Maximum \\
\hline BMI $(\mathrm{Kg} / \mathrm{m} 2)$ & $25.2 \pm 4$ & 17.6 & 47.3 \\
\hline Number of Junk food meals per week & $4 \pm 3$ & 0 & 21 \\
\hline \multirow[t]{2}{*}{ Number of water cups per day } & $6 \pm 4$ & 1 & 20 \\
\hline & & N. & $\%$ \\
\hline \multirow[t]{2}{*}{ BMI } & Normal weight & 203 & $50.75 \%$ \\
\hline & Overweight/Obese & 197 & $49.25 \%$ \\
\hline \multirow{5}{*}{ Junk food intake* } & No intake & 26 & $6.5 \%$ \\
\hline & Once weekly & 135 & $33.8 \%$ \\
\hline & Several times a week & 194 & $48.5 \%$ \\
\hline & Once daily & 25 & $6.3 \%$ \\
\hline & Several times a day & 20 & $5 \%$ \\
\hline History of weight control diet & Yes & 213 & $53.2 \%$ \\
\hline \multirow{4}{*}{ What made you follow diet } & Doctor & 21 & $9.8 \%$ \\
\hline & Friend & 14 & $6.6 \%$ \\
\hline & Media & 6 & $2.8 \%$ \\
\hline & A personal decision & 172 & $79 \%$ \\
\hline \multirow{2}{*}{ Satisfied with diet results } & Yes & 118 & $55.4 \%$ \\
\hline & No & 95 & $44.6 \%$ \\
\hline
\end{tabular}

*Junk food intake several times a week, means a number of weekly junk food meals more than once weekly and less than 7 time a week (ranged from 2-6 meals/week).

Table (3): Distribution of participants' choices on Body Figure Scale:

\begin{tabular}{|c|c|c|c|c|c|c|c|c|c|c|}
\hline & & Body $f$ & ures c & ices on 1 & sure $\mathbf{R a}$ & ng Scal & (FRS) & & & \\
\hline Body Image co & cept & Fig 1 & Fig 2 & Fig 3 & Fig 4 & Fig 5 & Fig 6 & Fig 7 & Fig 8 & Fig 9 \\
\hline Feeling body & $\mathrm{N}$ & 7 & 49 & 96 & 126 & 82 & 24 & 9 & 6 & 1 \\
\hline figure & $\%$ & 1.8 & 12.3 & 24.0 & 31.5 & 20.5 & 6 & 2.3 & 1.5 & 0.3 \\
\hline Ideal Body & $\mathrm{N}$. & 5 & 89 & 152 & 113 & 39 & 2 & 0 & 0 & 0 \\
\hline Figure & $\%$ & 1.3 & 22.3 & 38 & 28.3 & 9.8 & 0.5 & 0 & 0 & 0 \\
\hline Other gender & $\mathrm{N}$. & 3 & 42 & 124 & 182 & 45 & 3 & 1 & 0 & 0 \\
\hline ideal body figure & $\%$ & 0.8 & 10.5 & 31 & 45.5 & 11.3 & 0.8 & 0.3 & 0 & 0 \\
\hline Same sex ideal & N. & 9 & 51 & 132 & 146 & 60 & 1 & 1 & 0 & 0 \\
\hline body figure & $\%$ & 2.3 & 12.8 & 33 & 36.5 & 15.0 & 0.3 & 0.3 & 0 & 0 \\
\hline
\end{tabular}

Table (4): Distribution of body figures choices of the participants by their gender:

\begin{tabular}{|c|l|c|c|}
\hline \multicolumn{2}{|c|}{ Characteristics } & Other gender ideal body figure & Same sex ideal body figure for opposite sex \\
\hline \multirow{3}{*}{ Gender } & Males & $3.8 \pm 0.9$ & $3.8 \pm 1.2$ \\
\cline { 2 - 4 } & Females & $3.5 \pm 0.8$ & $3.3 \pm 0.9$ \\
\cline { 2 - 4 } & P value & $<\mathbf{0 . 0 0 1} *$ & $<\mathbf{0 . 0 0 1} *$ \\
\hline
\end{tabular}

\section{* Student $t$ test}

Table (5): Participants` body image assessment using Feeling-Actual Index (FAI):

\begin{tabular}{|l|l|l|l|}
\hline \multicolumn{2}{|c|}{ N.=400 } \\
\hline \multirow{2}{*}{ Feeling-Actual Index (FAI) scores } & \multicolumn{1}{|c|}{ Mean \pm SD } & \multicolumn{1}{c|}{ Minimum } & \multicolumn{1}{c|}{ Maximum } \\
\cline { 2 - 4 } & $-0.6 \pm 0.7$ & -2 & 1 \\
\hline \multirow{3}{*}{ Body image estimation } & Right body image estimation & N. & $\%$ \\
\cline { 2 - 4 } & Body image underestimation & 181 & $45.25 \%$ \\
\cline { 2 - 4 } & Body image overestimation & 5 & $53.5 \%$ \\
\hline
\end{tabular}


Table (6): relation between participants' body image assessment (FAI score) and their different characteristics:

\begin{tabular}{|c|c|c|c|c|}
\hline & & \multicolumn{3}{|c|}{ FAI score } \\
\hline \multicolumn{2}{|c|}{ Characteristics } & Mean & SD & P-value \\
\hline \multirow{2}{*}{ Gender } & Males & -0.59 & 0.69 & \multirow[b]{2}{*}{$0.54 *$} \\
\hline & Females & -0.61 & 0.63 & \\
\hline \multirow{2}{*}{ Nationality } & Egyptians & -0.6 & 0.64 & \multirow{2}{*}{$0.82 *$} \\
\hline & Other nationalities & -0.59 & 0.7 & \\
\hline \multirow{2}{*}{ Residence } & Cairo & -0.6 & 0.65 & \multirow{2}{*}{$0.86^{*}$} \\
\hline & Other & -0.62 & 0.66 & \\
\hline \multirow{4}{*}{ Marital status } & Single & -.058 & 0.64 & \multirow{4}{*}{$0.17 * *$} \\
\hline & Engaged & -0.83 & 0.70 & \\
\hline & Married & -0.85 & 0.89 & \\
\hline & Divorced & -1 & 0 & \\
\hline \multirow{4}{*}{$\begin{array}{l}\text { Living during scholar } \\
\text { year }\end{array}$} & With family & -0.64 & 0.68 & \multirow{4}{*}{$0.18 * *$} \\
\hline & With relatives & -0.36 & 0.50 & \\
\hline & With friends/Hostel & -0.48 & 0.54 & \\
\hline & Alone & -0.52 & 0.58 & \\
\hline \multirow{2}{*}{ University } & Ain shams & -0.58 & 0.66 & \multirow{2}{*}{$0.58^{*}$} \\
\hline & MUST & -0.62 & 0.64 & \\
\hline \multirow{2}{*}{ Faculty } & Medicine & -0.54 & 0.64 & \multirow{2}{*}{$0.058 *$} \\
\hline & Others & -0.66 & 0.66 & \\
\hline \multirow{2}{*}{ BMI } & Normal weight & 0.20 & 0.44 & \multirow{2}{*}{$<0.001 *$} \\
\hline & Overweight/Obese & -1.48 & 0.74 & \\
\hline \multirow{2}{*}{$\begin{array}{l}\text { Regular physical } \\
\text { activity }\end{array}$} & Yes & -0.55 & 0.67 & \multirow{2}{*}{$0.13 *$} \\
\hline & No & -0.64 & 0.65 & \\
\hline \multirow{2}{*}{$\begin{array}{l}\text { History of weight } \\
\text { control diet }\end{array}$} & Yes & -0.62 & 0.67 & \multirow{2}{*}{$0.51 *$} \\
\hline & No & -0.58 & 0.62 & \\
\hline \multirow{2}{*}{$\begin{array}{l}\text { Satisfied with diet } \\
\text { results }\end{array}$} & Yes & -0.56 & 0.65 & \multirow{2}{*}{$0.08 *$} \\
\hline & $\mathrm{No}$ & -0.74 & 0.69 & \\
\hline
\end{tabular}

*Mann- Whitney test $* *$ Kruskal-Wallis test

Table (7): Participants` body image satisfaction by feeling ideal discrepancy index (FID):

\begin{tabular}{|c|c|c|c|}
\hline \multirow{3}{*}{$\begin{array}{l}\text { Feeling-Ideal Discrepancy (FID) } \\
\text { scores }\end{array}$} & \multirow[b]{2}{*}{ Mean \pm SD } & \multicolumn{2}{|l|}{$\mathrm{N} .=400$} \\
\hline & & \multirow{2}{*}{ Minimum } & Maximum \\
\hline & $0.7 \pm 1.2$ & & 5 \\
\hline & & $\mathbf{N}$. & $\%$ \\
\hline \multirow{3}{*}{ Body image satisfaction } & Satisfied with current body image & 123 & $30.75 \%$ \\
\hline & Desire to lose weight & 221 & $55.25 \%$ \\
\hline & Desire to gain weight & 56 & $14 \%$ \\
\hline
\end{tabular}


Table (8): relation between participants' body image satisfaction and their characteristics:

\begin{tabular}{|c|c|c|c|c|}
\hline \multirow{2}{*}{\multicolumn{2}{|c|}{ Characteristics }} & \multicolumn{3}{|c|}{ FID score } \\
\hline & & \multirow{2}{*}{$\begin{array}{l}\text { Mean } \\
0.5\end{array}$} & \multirow{2}{*}{ SD } & P-value \\
\hline \multirow{2}{*}{ Gender } & Males & & & \multirow[b]{2}{*}{$<0.001 *$} \\
\hline & Females & 0.8 & 1.2 & \\
\hline \multirow{2}{*}{ Nationality } & Egyptians & 0.7 & 1.2 & \multirow{2}{*}{$0.29 *$} \\
\hline & Other nationalities & 0.5 & 1.1 & \\
\hline \multirow{2}{*}{ Residence } & Cairo & 0.7 & 1.2 & \multirow{2}{*}{$0.55^{*}$} \\
\hline & Other & 0.8 & 1.3 & \\
\hline \multirow{4}{*}{ Marital status } & Single & 0.6 & 1.2 & \multirow{4}{*}{$0.02 * *$} \\
\hline & Engaged & 1.1 & 1.3 & \\
\hline & Married & 1.6 & 1 & \\
\hline & Divorced & 1.5 & 0.6 & \\
\hline \multirow{4}{*}{ Living during scholar year } & With family & 0.7 & 1.1 & \multirow{4}{*}{$0.09 *$} \\
\hline & With relatives & 1.2 & 1.7 & \\
\hline & With friends/Hostel & 0.5 & 1.2 & \\
\hline & Alone & 0.2 & 1.1 & \\
\hline \multirow{2}{*}{ Father`s education } & Below university degree & 0.5 & 1 & \multirow{2}{*}{$0.26 *$} \\
\hline & University/post graduate & 0.7 & 1.2 & \\
\hline \multirow{2}{*}{ Mother`s education } & Below university degree & 0.6 & 1.1 & \multirow{2}{*}{$0.57 *$} \\
\hline & University/post graduate & 0.7 & 1.2 & \\
\hline \multirow{2}{*}{ University } & Ain shams & 0.65 & 1.2 & \\
\hline & MUST & 0.8 & 1.2 & \\
\hline \multirow{2}{*}{ Faculty } & Medicine & 0.7 & 1.2 & \multirow{2}{*}{$0.2 *$} \\
\hline & Others & 0.6 & 1.1 & \\
\hline \multirow{2}{*}{ BMI } & Normal weight & 0.2 & 1.2 & \multirow{2}{*}{$<0.001 *$} \\
\hline & Overweight/Obese & 1.1 & 1.2 & \\
\hline \multirow{2}{*}{ Regular physical activity } & Yes & 0.7 & 1.2 & \multirow{2}{*}{0.73} \\
\hline & No & 0.7 & 1.2 & \\
\hline \multirow{2}{*}{ History of weight control diet } & Yes & 1 & 1.2 & \multirow{2}{*}{$<0.001 * *$} \\
\hline & No & 0.3 & 1 & \\
\hline \multirow{2}{*}{ Satisfied with diet results } & Yes & 0.9 & 1.2 & \multirow{2}{*}{$<0.001 * *$} \\
\hline & No & 1.3 & 1.2 & \\
\hline
\end{tabular}

*Mann- Whitney test. **Kruskal-Wallis test 
Table (9): Relation between participants' body image concerns, and their characteristics:

\begin{tabular}{|c|c|c|c|c|c|c|}
\hline \multirow{3}{*}{\multicolumn{2}{|c|}{ Characteristic }} & \multicolumn{5}{|c|}{ Body image concerns } \\
\hline & & \multicolumn{2}{|c|}{$\begin{array}{l}\text { Positive body } \\
\text { image }\end{array}$} & \multicolumn{2}{|c|}{$\begin{array}{c}\text { Negative body } \\
\text { image }\end{array}$} & \multirow[t]{2}{*}{ p-value* } \\
\hline & & N. & $\%$ & N. & $\%$ & \\
\hline \multirow{2}{*}{ Gender } & Male & 126 & $86.3 \%$ & 20 & $13.7 \%$ & \multirow{2}{*}{0.2} \\
\hline & Female & 207 & $81.5 \%$ & 47 & $18.5 \%$ & \\
\hline \multirow{2}{*}{ Nationality } & Egyptians & 280 & $81.6 \%$ & 63 & $18.4 \%$ & \multirow{2}{*}{0.03} \\
\hline & Other nationalities & 53 & $93 \%$ & 4 & $7 \%$ & \\
\hline \multirow{2}{*}{ Residence } & Cairo & 299 & $85.4 \%$ & 51 & $14.6 \%$ & \multirow{2}{*}{0.002} \\
\hline & Other & 34 & $68 \%$ & 16 & $32 \%$ & \\
\hline \multirow{4}{*}{ Marital status } & Single & 308 & $83 \%$ & 63 & $17.0 \%$ & \multirow{4}{*}{$0.65 \mathrm{FE}$} \\
\hline & Engaged & 16 & $88.9 \%$ & 2 & $11.1 \%$ & \\
\hline & Married & 5 & $71.4 \%$ & 2 & $28.6 \%$ & \\
\hline & Divorced & 3 & $75 \%$ & 1 & $25 \%$ & \\
\hline \multirow{4}{*}{$\begin{array}{l}\text { Living during } \\
\text { scholar year }\end{array}$} & With family & 255 & $82.3 \%$ & 55 & $17.7 \%$ & \multirow{4}{*}{$\begin{array}{l}0.2 \\
\text { FE }\end{array}$} \\
\hline & With relatives & 8 & $72.7 \%$ & 3 & $27.3 \%$ & \\
\hline & With friends/Hostel & 45 & $83.3 \%$ & 9 & $16.7 \%$ & \\
\hline & Alone & 24 & $96 \%$ & 1 & $4 \%$ & \\
\hline \multirow{2}{*}{ Father`s education } & Below university degree & 33 & $68.8 \%$ & 15 & $31.3 \%$ & \multirow{2}{*}{0.004} \\
\hline & University/post graduate & 300 & $85.2 \%$ & 52 & $14.8 \%$ & \\
\hline \multirow{2}{*}{ Mother`s education } & Below university degree & 61 & $73.5 \%$ & 22 & $26.5 \%$ & \multirow{2}{*}{0.008} \\
\hline & University/post graduate & 272 & $85.8 \%$ & 45 & $14.2 \%$ & \\
\hline \multirow{2}{*}{ University } & Ain Shams & 158 & $47.4 \%$ & 48 & $71.6 \%$ & \multirow{2}{*}{$<0.001$} \\
\hline & MUST & 175 & $52.6 \%$ & 19 & $28.4 \%$ & \\
\hline \multirow{2}{*}{ Faculty } & Medicine & 171 & $51.3 \%$ & 33 & $49.2 \%$ & \multirow{2}{*}{0.7} \\
\hline & Other & 162 & $48.7 \%$ & 34 & $50.8 \%$ & \\
\hline \multirow{2}{*}{ BMI } & Normal weight & 170 & $83.7 \%$ & 33 & $16.2 \%$ & \multirow{2}{*}{0.02} \\
\hline & Overweight/obese & 134 & $68 \%$ & 34 & $17.3 \%$ & \\
\hline \multirow{2}{*}{$\begin{array}{l}\text { Regular physical } \\
\text { activity }\end{array}$} & Yes & 145 & $87.3 \%$ & 21 & $12.7 \%$ & \multirow{2}{*}{0.06} \\
\hline & No & 188 & $80.3 \%$ & 46 & $19.7 \%$ & \\
\hline \multirow{2}{*}{$\begin{array}{l}\text { History of weight } \\
\text { control diet }\end{array}$} & Yes & 176 & $82.6 \%$ & 37 & $17.4 \%$ & \multirow{2}{*}{0.72} \\
\hline & No & 157 & $84.0 \%$ & 30 & $16.0 \%$ & \\
\hline Satisfied with diet & Yes & 120 & $85.1 \%$ & 21 & $14.9 \%$ & 023 \\
\hline results & No & 59 & $78.7 \%$ & 16 & $21.3 \%$ & 0.23 \\
\hline
\end{tabular}

*Chi- square test

FE: Fisher exact test of significance

Table (10): Self-esteem score of the participants and its correlation with FAI, FID and BIC scores:

\begin{tabular}{|l|c|c|c|c|}
\hline & Mean & SD & r* & P value \\
\hline Self-esteem score & 20.36 & 4.47 & & \\
\hline FAI index & & & & \\
\hline FID index & -0.6 & 0.7 & -0.004 & 0.93 \\
\hline Body image concerns scale & 0.7 & 1.2 & 0.03 & 0.61 \\
\hline
\end{tabular}

*Spearman correlation coefficient $\quad * *$ Pearson correlation coefficient

shows that females had significantly higher FID score than males (p value < 0.05). Married participants had higher scores ( $\mathrm{p}$ value $<0.05)$. Overweight/obese participants had significantly higher scores than normal weight ( $p$ value $<0.05$ ). Participants with history of weight control diet had higher scores ( $\mathrm{p}$ value $<0.05$ ). Those not satisfied with diet results had higher scores ( $\mathrm{p}$ value $<0.05)$. Other participants' characteristics had no significant relation with FID score.

When participants are classified according to their body image concerns, $333(83.2 \%)$ participants had positive 
concerns and 67 (16.8\%) had negative body image concerns (figure 2). Regarding relation between participants' body image concerns and their different characteristics, (table 9) shows that there is a statistically significant relation between body image concerns and each of participants nationality, residence, father and mother education level, university and BMI ( $\mathrm{p}$ value <0.05). Non-Egyptian participants, participants living in Cairo, participants with highly educated fathers and mothers, Must University students and normal weight participants had higher percent of positive body image concerns than others.

Participants are divided according to their self-esteem into two groups, 366 (91.5\%) participants with high selfesteem (score $\geq 15$ ) and $34(8.5 \%)$ participants with low self-esteem (score $<15$ ) (figure 3). Self-esteem score of participants` ranged from 6 to 30, with the mean of $20.4 \pm 4.47$. There was a statistically significant positive correlation between self-esteem score and body image concerns scale ( $\mathrm{p}$ value $<0.05$ ) (table 10).
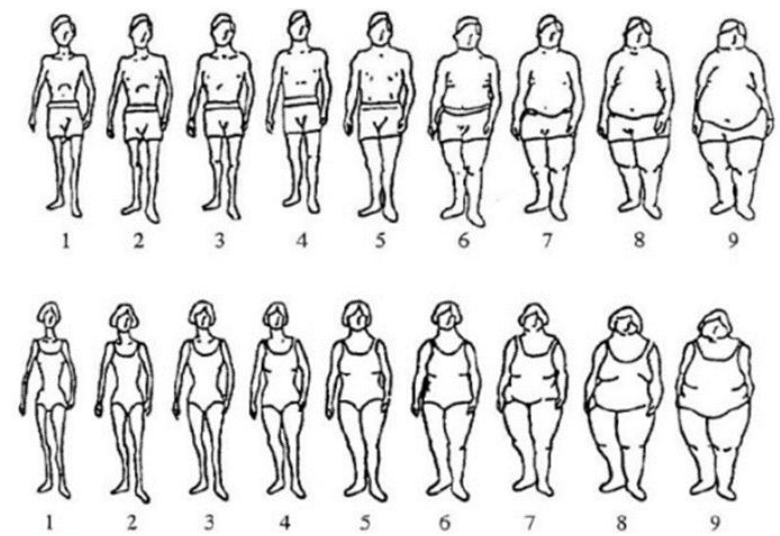

Figure (1): Body figure rating scale

\section{Discussion:}

University students represent a vulnerable group to different pressures to follow perceived culturally accepted standards of ideal physical appearance. They are at a critical developmental age

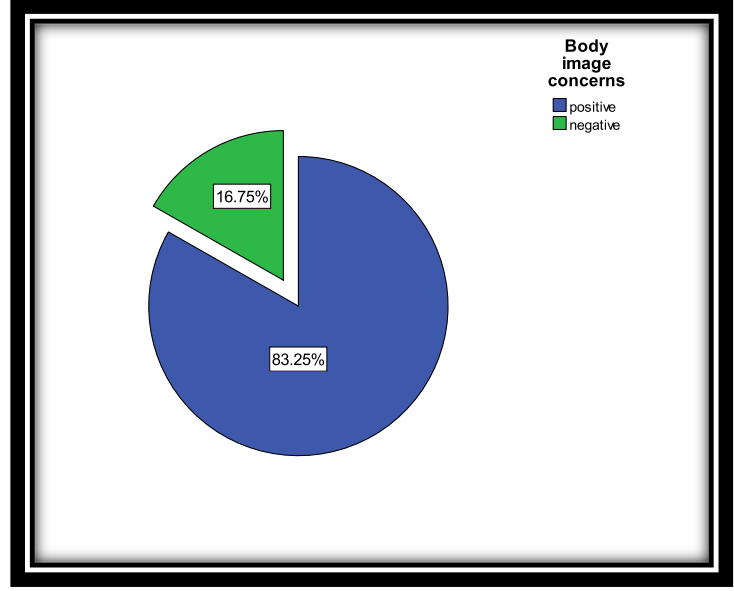

Figure 2: Distribution of participants according to body image concerns

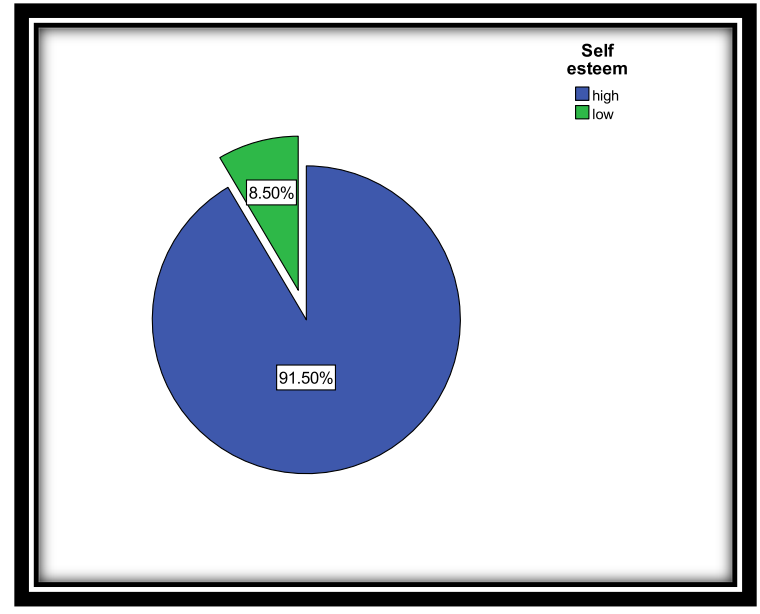

Figure 3: Distribution of participants according to their self esteem

susceptible to many pressures that may affect their self-esteem. ${ }^{9}$

The current study was carried out in a governmental and a private university to help in understanding the university students perceptions towards the different concepts of body image and its association with socio-demographic characteristics, nutritional and physical activity habits and to determine the effects of misperception on their selfesteem. The current study used the Figure Rating Scale the most widely preferred tool for the assessment of body size and weight-related components of body image in addition to the body image concerns (BIC) validated scale used to measure body image concerns (positive or negative body image). ${ }^{17-20}$ 
Using the Figure Rating Scale $55.5 \%$ of students selected figures number 3,4 illustrating normal weight figures to represent how they currently feel that their bodies look like. Similarly, two thirds of students $(66.3 \%)$ selected average weight figures (figures 3 and 4) to represent their ideal body status. Moreover $38 \%$ of students selected figure 3 as ideal body figure and figure 4 was the most frequently selected figure for opposite gender and same gender ideal body accounting for $45.5 \% \%$ and $36.5 \%$ respectively. This shows that both genders prefer ideals of normal weight which may be due to the increased exposure to westernized ideals of beauty through media ${ }^{17}$ These results conform with Zaccagni et al. 2014 study that assessed body image perception and the degree of dissatisfaction among a sample of Italian university students and showing that figure 3 was the most frequently selected as opposite sex ideal. ${ }^{3}$ Similarly, in a study involving Arab female university students from Egypt, Bahrain, Jordan, Oman and Syria results showed that they preferred average weight figures to represent their ideal body figures for their gender and for opposite sex. ${ }^{11}$

However, these results are in disagreement with a study conducted on female students aged 18-35 years from Tabriz, Iran, in which most of the participants selected the thinnest figure as their desirable or ideal body image. ${ }^{21}$ Moreover, the results disagree with the results of a previous study that was conducted in Kenya, in 2013 in which most of the participants preferred obese figures to represent their ideal or desirable body figures for their own gender and the other gender. ${ }^{22}$

This may be explained by the fact that body image perception is strongly influenced by families, communities, and society. In some communities in subSaharan Africa excess body weight has generally been associated with wealth and health and considered a desirable attribute. ${ }^{23-24}$ However, urbanization and increasing exposure to westernized media portraying thin ideals have extreme effects on body image perceptions in developing countries especially among adolescents and young adults. $^{25}$

In the current study female students were asked to select the female figure that they believed to represent the opposite sex ideal for men. While male students were asked to choose the male figure they felt would be the opposite sex ideal for women. Female students felt the opposite sex will desire a thinner ideal (3.3 \pm 0.9$)$ than it actually does $(3.8 \pm 0.9)$. However, male selected a larger figure for same and opposite sex ideals. These findings agree with many researches. ${ }^{3,26}$ These results suggest that men perceive thin male figures as lack of muscles, while women perceive larger women figures as excess weight or excess fat mass. This may be due to the fact that Stunkard silhouette figures are uni-dimensional and it is hard to distinguish between increase in their dimensions due to excess muscle or excess fat. ${ }^{3}$

Using the Feeling Actual Index (FAI) the current study revealed that more than half $(53.5 \%)$ of the student underestimated their body image, $45.2 \%$ and only $1.25 \%$ of them, had correct and overestimation of their body image respectively. Mean \pm SD FAI was 0.6 \pm 0.7 . Negative values indicate underestimation. Both males and females in the present study had negative values with no statistically significant difference. This indicates that in the current study both sexes tend to underestimate their actual weight. These findings contrast with an Italian study reporting positive mean FAI values in females and negative in males, indicating that women tend to overestimate their weight while men tend to underestimate it. ${ }^{18}$ The current study results disagree with Sánchez-Villegas et al., study 
revealing that overall, women classified themselves statistically significantly better than men being $57.6 \%$ vs. $32.7 \%$. respectively $(\mathrm{P}<0.001)^{26}$. The current study results disagree with a study of university students in Navarra, Spain showing that $43.03 \%$ of students, overestimated their body image $(10.65 \%$ in males and $59.69 \%$ in females) while $10.20 \%$ and $46.75 \%$ of students underestimated and had concordance between BMI and body image perception respectively. ${ }^{27}$

In the current study the mean \pm SD of FAI were $0.20 \pm 0.44$ and $-1.48 \pm 0.74$ $(\mathrm{p}<0.001)$ in normal weight and overweightlobese respectively. This indicates that overweightlobese tend to underestimate their actual weight in contrast normal weight study participants tend to overestimate it. This finding agrees with Ettarh et al., study in Kenya showing that more than half of the population who were overweight or obese underestimated their weight. ${ }^{22}$

It should be mentioned that correct estimation of actual body weight indicates that people are aware of their current weight status, this is necessary for optimal body weight management and prevention of weight-related diseases. $^{3,22}$ On the other hand, underestimating the actual body weight status may lead to paying less attention to nutritional status and dietary habits as a result of lack of perception of having a weight problem. Consequently, these individuals will underestimate the risky consequences of the extra weight ${ }^{22,28}$. Thus, the results of the current study highlight that the majority of studied students underestimated their current body size. This may end in continuing to maintain a high BMI. As the overweight and obesity are prevalent among our study participants as a result they will have a high risk for many noncommunicable diseases.

Many studies have shown that many socio-cultural factors affect dissatisfaction with body size. ${ }^{28}$ In the current study only $30.75 \%$ of the university students were satisfied with their current body image, $55.25 \%$ and $14.0 \%$ want to lose weight and gain weight respectively. Both genders suffer from body image dissatisfaction and females show higher statistically significant desire to be thinner. The increase in FID was significantly higher in engaged, married and divorced than single, overweight/obese than normal weight study participants. University students reporting a history of weight control by diet and non- satisfactory results of the weight management diet had significantly higher FID. These findings conform with many studies showing that women have higher dissatisfaction with their body than men. Similarly, there is increase in FID indicating higher body dissatisfaction among overweight and obese participants with higher BMI. As a result, individuals who feel body dissatisfaction are more likely to participate in behaviors to fight the discomfort such as weight reduction diet. ${ }^{3,28,30,19}$

Using the body image concern scale getting 67 and above means positive body image concerns and as the score increases this means that the person feels comfortable and happy with his/her body looks and is less likely to be affected by unrealistic images in the media and societal pressures to look in a certain way. In the current study $333(83.2 \%)$ of study participants had positive concerns and 67 (16.8\%) had negative body image concerns. Non-Egyptian participants, participants living in Cairo, participants with highly educated fathers and mothers, MUST university students and normal weight participants had higher percent of positive body image concerns than others. ( $p$ value $<0.05$ ).

On the other hand, these findings contrast with Khalaf et al., 2015 study showing that higher education and socioeconomic status of the parents were 
associated with higher body image dissatisfaction among Saudi female university students. ${ }^{20}$

Non-Egyptian participants had positive body image concerns more than the Egyptians but overall, both Egyptians and non-Egyptians had positive body image taking into consideration that most of the non-Egyptian participants in the current study were Syrians and Palestinians. This comes in agreement with a previous study conducted among Arab adolescents and female university students, which stated that being slightly overweight was more acceptable in Syria and Jordan, than in Egypt $(\mathrm{P}<0.001) .{ }^{19}$

The current study results showed that 91.5\% of the participants had high selfesteem. According to the Rosenberg scale of self-esteem and there was a statistically significant positive correlation between self- esteem score and body image concerns scale ( $\mathrm{p}$ value $<0.05$ ). This agree with ALAhmari et al., study conducted among female Saudi students showing that the prevalence of low self-esteem was only $6.1 \%$ among studied students. ${ }^{31}$ It is important to note that the variables associated with body image perception, satisfaction and selfesteem are complex and are similar in many Middle East countries. For instance, perceived body image and a desire to be thinner were strongly related to body image dissatisfaction, the association between higher BMI levels and higher dissatisfaction and lower positive body image concerns. 7,19

Due to the scarcity of studies in the Eastern Mediterranean region on the body image perception and on associated socio- cultural factors as well as consequences of distorted and dissatisfying body image as depression, self-esteem and eating disorders. Further studies are required especially among young adolescents as they may be in a critical phase of their development in order to understand the perceptual dimension of BI and perceived body weight with regards to actual body weight among them.

In addition, the current study revealed that more than half $(53.5 \%)$ of the student underestimated their body image. Consequently, innovative strategies are required to ensure better appreciation of the actual body size and understanding of the health consequences of overweight and obesity and to reverse the acceptance for larger body size. Designing tailored effective dietary and lifestyle interventions are needed to address the epidemic of overweight and obesity especially among youth.

\section{Limitations:}

The study included only two universities which may not be representative for university students in Egypt. As a crosssectional survey, the current study revealed the prevalence and the underlying risk factors. However, the directions of effects couldn't be ascertained (findings are associations not causations). The used Body Figure Rating Scale "Stunkard Scale", is a one dimensional scale, it only shows the increase in body figures from thinnest to fattest, without demonstrating that increase is due to increased fats or increased muscle bulk. Also it doesn't show the different body shapes and body shape defects, Thus, no idea about the participants`satisfaction with their own body shapes or defects. The used scales were based on self-reporting measures that is completely dependent on the cooperation and frankness of the subjects.

\section{References}

1. Grogan S. Body image: understanding body dissatisfaction in men, women and children. Abingdon, UK: Taylor \& Francis; 2016. P. 1-9.

2. Tylka T. L. and Wood-Barcalow N. L. What is and what is not positive body image? Conceptual foundations and construct definition. Body image. 2015, 14, 118-129. 
3. Zaccagni L., Masotti S., Donati R., Mazzoni G. and Gualdi-Russo E. Body image and weight perceptions in relation to actual measurements by means of a new index and level of physical activity in Italian university students. Journal of translational medicine. 2014, 12(1), p.42

4. Bucchianeri M. M., Arikian A. J., Hannan P. J., Eisenberg M. E. and NeumarkSztainer D. Body dissatisfaction from adolescence to young adulthood: Findings from a 10-year longitudinal study. Body Image. 2013 10(1), 1-7.

5. Smink F. R., Van Hoeken D., Hoek H. W. Epidemiology of eating disorders: incidence, prevalence and mortality rates. Current psychiatry reports. 2012, 14(4), 406414.

6. Stunkard A.J., Sorensen T., Schulsinger F. Use of the Danish Adoption Register for the study of obesity and thinness. Res Publ Assoc Res Nerv Ment Dis. 1983; 60:115-20. 7. Kabir Y., Zafar T.A., Waslien C. Relationship between perceived body image and recorded body mass index among Kuwaiti female university students. Women Health. 2013; 53(7):693-705.

8. Ozmen D., Ozmen E., Ergin D., Cetinkaya A.C., Sen N., Dundar P.E., Taskin E.O. The association of self-esteem, depression and body satisfaction with obesity among Turkish adolescents. BMC Public Health. 2007 May 16;7:80.

9. Arnett J. J. Emerging adulthood: A theory of development from the late teens through the twenties. Am Psychol. 2000, 55, 469-480.

10. Musaiger A. O. and Al-Mannai M. Association between exposure to media and body weight concern among female university students in five Arab countries: a preliminary cross-cultural study. J Biosoc Sci. 2014, 46(2), 240-247.

11. Musaiger A. O., bin Zaal A. A. and D'Souza R. Body weight perception among adolescents in Dubai, United Arab Emirates. Nutr Hosp. 2012, 27(6), 1966-1972. http://dx.doi.org/10.3305/nh.2012.27.6.583 0.

12. Dean A.G., Arner T.G., Sunki G.G., Friedman R., Lantinga M., Sangam S., et al. Epi InfoTM, a database and statistics program for public health professionals. CDC, Atlanta, GA, USA, 2011.
13.Abdul-Naby S. The Relationships among Body Image, Self-esteem and Depression in a Sample of University Students. Journal of Mental researches of Monofeya University. 2008, 1, 187-235.

14.Cooper P., Taylor M. J., Cooper Z. and Fairburn C. G. The development and validation of the body shape questionnaire. Int J Eat Disord. 1987, 6(4), 498-506.

15. Rosenberg M., Schooler C. and Schoenbach, C. Self-esteem and adolescent problems: Modeling reciprocal effects. American sociological review. 1989, 10041018.

16. WHO, Expert Consultation "Appropriate body-mass index for Asian populations and its implications for policy and intervention strategies." Lancet (London, England). 2004, 363, no. 9403: 157

17. Tehard B., Van Liere M.J., Com Nougué C., Clavel-Chapelon F. Anthropometric measurements and body silhouette of women: Validity and perception. Journal of the American Dietetic Association. 2002, 102(12), 1779-1784.

18. Swami V., Salem N., Furnham A., Tovée M.J. Initial examination of the validity and reliability of the female photographic figure rating scale for body image assessment. Personality and Individual Differences. 2008. Available online at: <www.sciencedirect.com>

19. Musaiger A. Body weight concern among female university students in five Arab countries--a preliminary cross-cultural study. Ann Agric Environ Med.2015;22(2):349-52.

20. Khalaf A., Westergren A., Berggren V., Ekblom Ö., Al-Hazzaa H.M. Perceived and Ideal Body Image in Young Women in South Western Saudi Arabia. J Obes.2015;2015:697163.

21. Alipour B., Abbasalizad F. M., Dehghan P., Alipour M. Body image perception and its association with body mass index and nutrient intakes among female college students aged 18-35 years from Tabriz, Iran. Eat Weight Disord. 2015 Dec;20(4):465-71. doi: 10.1007/s40519-015-0184-1. Epub 2015 Feb 21. PubMed PMID: 25701442.

22. Ettarh R., Van de Vijver S., Oti S., Kyobutungi C. Overweight, obesity, andperception of body image among slum residents in Nairobi, Kenya, 2008-2009. Prev Chronic Dis. 2013 Dec 19;10:E212. 
23. Duda R.B., Jumah N.A., Hill A.G., Seffah J., Biritwum R. Assessment of the ideal body image of women in Accra, Ghana. Trop Doct 2007;37(4):241-4 10.

24. Swami V., Frederick D.A., Aavik T., Alcalay L., Allik J., Anderson D., et al. The attractive female body weight and female body dissatisfaction in 26 countries across 10 world regions: results of the International Body Project I. Pers Soc Psychol Bull 2010;36(3):309-25.

25. Bibiloni M. D., Coll J. L., Pich J., Pons A., Tur J. A. Body image satisfaction and weight concerns among a Mediterranean adult population. BMC Public Health. 2017; 17;39 PMID: 28061761

26. Sánchez-Villegas A., Madrigal H., Martínez-González M.A., Kearney J., Gibney M.J., de Irala J., Martínez J.A. Perception of body image as indicator of weight status in the European union. J Hum Nutr Diet. 2001 Apr;14(2):93-102.

27. Soto Ruiz M.N., Marin Fernández B., Aguinaga Ontoso I., Guillén-Grima F., Serrano Mozó I., Canga Armayor N., Hermoso de Mendoza Cantón J., Stock C.,
Kraemer A, Annan J. Analysis of body image perception of university students in Navarra. Nutr Hosp. 2015 May 1;31(5):2269-75. PubMed PMID: 25929403. 28. Kruger J., Lee C.D., Ainsworth B.E., Macera C.A. Body size satisfaction and physical activity levels among men and women. Obesity. 2008;16(8):1976-1979.

29. Kakeshita I.S. and de Sousa Almeida S. Relationship between body mass index and self-perception among university students. Rev Saude Publica. 2006;40(3):497-594.

30. Carraça E.V., Silva M.N., Markland D., Vieira P.N., Minderico C.S., Sardinha L.B., Teixeira P.J. Body image change and improved eating self-regulation in a weight management intervention in women. Int $\mathbf{J}$ Behav Nutr Phys Act. 2011;8:75.

31. ALAhmari T., Alomar A.Z., ALBeeybe J., Asiri N., ALAjaji R., ALMasoud R., AlHazzaa H.M. Associations of self-esteem with body mass index and body image among Saudi college-age females. Eat Weight Disord. 2017 Dec 27. doi: 32.10.1007/s40519-017-0471-0. [Epub ahead of print] PubMed PMID: 29282654. 